\section{An elasto-plastic contact model for conformal contacts between cylinders}

\author{
Jianqiao Hu ${ }^{1,2}$, Fuhai $\mathrm{Gao}^{3}$, Xiaoming Liu ${ }^{1,2}$ (1) and \\ Yueguang $\mathrm{Wei}^{4}$
}

Proc IMechE Part J:

J Engineering Tribology

0(0) I-9

(C) IMechE 2019

Article reuse guidelines:

sagepub.com/journals-permissions DOI: |0.1 |77//350650||989646|

journals.sagepub.com/home/pij

\begin{abstract}
In this paper, we present a new model for the elasto-plastic conformal contact between cylinders. By using finite element simulation, it is observed that the transition of elasto-plastic contact in conformal case is different from the transition in Hertz contact. The plastic contact of conformal case can be physically represented by the plastic-Winkler model. The new model provides an explicit solution for the normal force-displacement relation of elasto-plastic conformal contact. Compared to other elastic contact models of conformal contact, the advantages of our model are: (I) it can be used for high load contact and (2) plasticity is taken into account by using the plastic-Winkler model. Our model, therefore, is capable of dealing with realistic engineering problem with high load and plasticity.
\end{abstract}

\title{
Keywords
}

Conformal contact, elasto-plastic transition, normal force-displacement relation, plastic-Winkler model

Date received: 24 July 2019; accepted: 29 November 2019

\section{Introduction}

Clearance in the cylindrical joints plays a critical role in the performance of a mechanical system. A proper description of cylindrical joints mechanical behaviors requires a conformal contact model between cylinders where, especially, the normal force-displacement (NFD) relation is provided. The need for the conformal contact model has captured considerable attention of a large number of researchers, and several theoretical and experimental works have been carried out to study the mechanical behavior of cylindrical joints with clearances. ${ }^{1-3}$

Theoretical studies of two-dimensional conformal contact have been carried out to find the proper NFD relation in the past decade. It started from Persson ${ }^{4}$ who provided a closed form for the conformal contact (details can be found in Appendix 1). However, the formulation of Persson model in equation (13) is extremely complex and the normal force cannot be obtained with the geometrical boundary conditions explicitly. This hinders the application of the model in engineering. By revisiting Persson's solution, Ciavarella and Decuzzi ${ }^{5}$ provided a closed form relation between the normal load and the contact angle in the case of elastic similarity. More general case with elastic dissimilarity has been further studied, ${ }^{6}$ and numerical analysis was performed to confirm the accuracy of the closed form results. More recently, Fang et al. ${ }^{7}$ numerically fitted the expression of the pressure distribution and further proposed a universal solution for the conformal contact. However, the provided NFD relation, which is crucial for solving the clearance contact problem, was still not explicit. As a result, the numerical iteration is required to evaluate the contact force which increases the numerical complexity in the computational analysis.

There are some explicit NFD models for dynamic analysis of cylindrical joints. One of them is Johnson model $^{8}$ : this model has a logarithmic expression of the NFD relationship, which imposes some mathematical and physical limitations on the contact analysis. Other models $^{9-11}$ (e.g. Goldsmith, Lankarani-Nikravesh, and Radzimovsky models) have other limitations. For example, by comparative assessments, Pereira et al. ${ }^{12,13}$ concluded that in the condition of conformal contact between cylinders, Goldsmith model and Lankarani-Nikravesh model are not appropriate for

\footnotetext{
'State Key Laboratory of Nonlinear Mechanics, Institute of Mechanics, Chinese Academy of Sciences, Beijing, PR China

${ }^{2}$ School of Engineering Science, University of Chinese Academy of Sciences, Beijing, PR China

${ }^{3}$ China Institute of Atomic Energy, Beijing, PR China

${ }^{4}$ College of Engineering, Peking University, Beijing, PR China
}

\section{Corresponding author:}

Xiaoming Liu, Chinese Academy of Sciences, Room 233, Building I, 4th Ring North I5, Beijing 100864, China.

Email: xiaomingliu@imech.ac.cn 
modeling contact with low clearance. Instead, the use of Johnson and Radzimovsky contact model is recommended. Then, Pereira et al. ${ }^{14}$ introduced an enhanced cylindrical contact model based on the Johnson contact model, and this model is still only suitable within the scope of Johnson model (for low contact loads). Liu et al. ${ }^{15,16}$ proposed a simplified model for cylindrical joints with clearances; however, this model is not self-consistent, the assumption of the stress distribution they used largely deviates from the conformal contact condition. Other numerical models are used to obtain NFD for conformal contact. Blanco-Lorenzo et al. ${ }^{17}$ studied the NFD under different conformity levels by carrying out a frictionless contact in the framework of finite element method (FEM). They demonstrated that the Hertzian distribution could not provide precise contact pressure when the contact angle is high. Boundary element method (BEM) has also been used for the analysis of conformal contact of spherical and cylindrical inclusions, ${ }^{18}$ in which the contact stress distribution was studied as a function of the material properties, contact geometry, and interfacial conditions. However, the accuracy of numerical results strongly depends on the selection of simulation parameters, and a unified NFD relation is obscure.

The NFD relation in these previous models has some limitations in common: (1) NFD relation is expressed in a form that penetration is the function of the contact force; however, in general (both in the experiments and simulations), penetration/displacement is an easier measurable quantity, and therefore an NFD where the contact force is a function of penetration is desired. (2) These models are supposed to be only available for simple elastic case, thus cannot be used for the contact with plasticity. (3) Some of these models which have a load limit for the clearance are not applicable for high loads.
In this paper, we aim to develop an explicit NFD relation for the conformal contact that is effective for the high load and plasticity without the loss of accuracy. The paper is organized as follows: in the next section, we firstly revisit the assumption of the Hertz pressure distribution for the conformal contact, we propose a new pressure distribution model and verified by FEM result. Then, a contact model with explicit NFD relation is proposed. The proposed model is compared with FEM results and other contact models. Next, we extend the model for elasto-plastic deformation, and the model results are compared with FEM simulation results. Details of other contact models and our FEM simulations can be found in Appendices 1 to 3 .

\section{Contact pressure distribution in conformal contact}

The conformal contact between two cylindrical bodies is illustrated in Figure 1(a). The geometric and material parameters are listed in the notation. In a previous model, ${ }^{7}$ the contact force distribution in the projection plane is expressed as

$$
p(r)=p_{0}\left(1-\frac{r^{2}}{a^{2}}\right)^{n}
$$

where $p_{0}$ is the max contact pressure, $a$ is the radius of the contact area, $r$ is the distance to the center of the contact, and $n$ is the pressure distribution exponent. The value of $n$ was numerically fitted by Fang et al. ${ }^{7}$ However, the exponent has a complicated expression so that an explicit NFD cannot be obtained.

Another contact force model for conformal contact is proposed by Steuermann (see Noble and Hussain ${ }^{19}$ ). The model provides the pressure distribution for the surface profile with the form of $\sum_{n} A_{n} r^{2 n}\left(A_{n}\right.$ and $n$
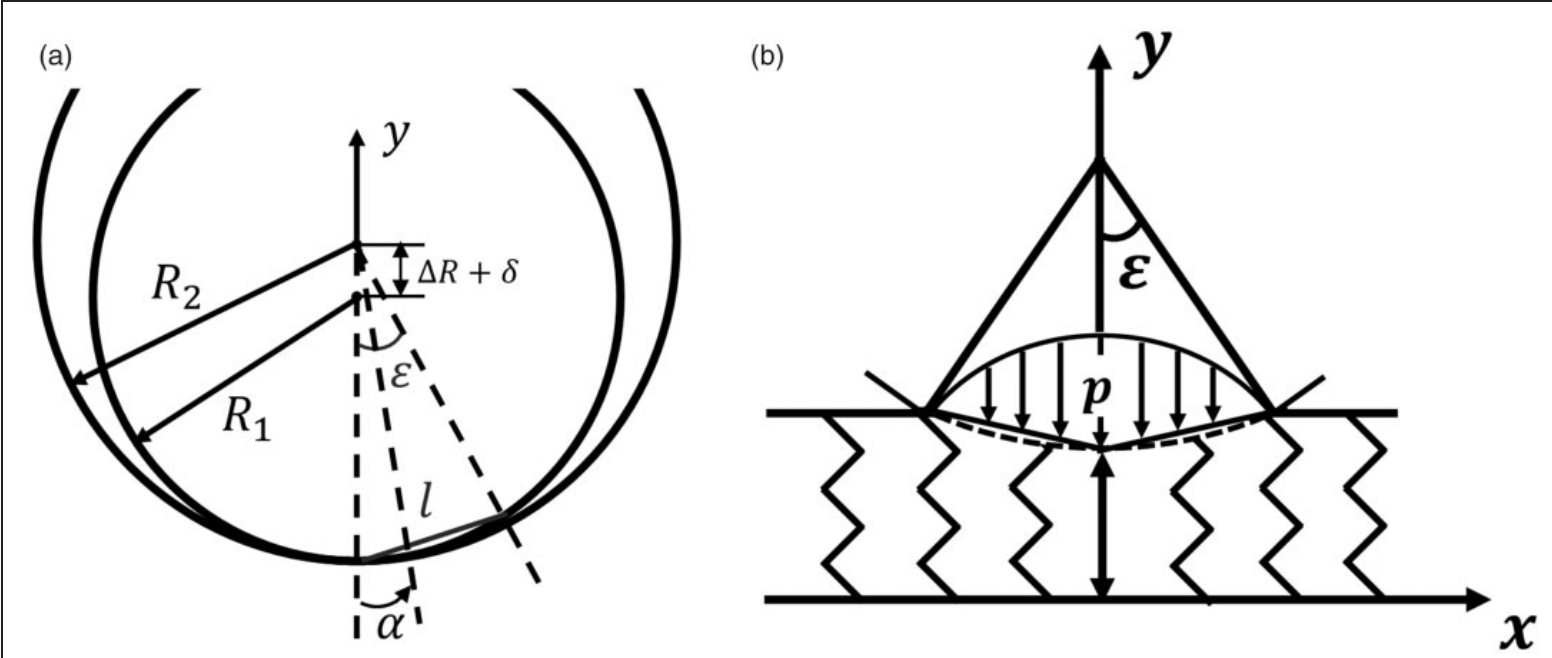

Figure I. (a) Schematic of the cylindrical joints with clearance. (b) Winkler contact model for joints with clearance. 
are fitting parameters depending on the contact profile). This model could be more accurate than that of Hertz because it includes high-order term of geometric profile. However, as pointed in contact mechanics by Johnson, ${ }^{8}$ the Steuermann model would also bring unacceptable errors for high contact angle $(\tan (\varepsilon / 2)>0.4$, where $\varepsilon$ is the semi-angle of contact).

Winkler elastic foundation model is another model for the conformal contact of cylindrical joints. ${ }^{15,16,20,21}$ In this model, as shown in Figure 1(b), the inner cylinder is assumed to be equivalent to a rigid wedge. Instead of using the projection plane as in the above mentioned models, ${ }^{7,19}$ the contact force model is built along the chord of the rigid wedge. Liu et al.'s model ${ }^{15,20}$ used Hertz distribution for the contact pressure, which has an elliptic form

$$
p_{y}=p_{0}\left(1-\frac{x^{2}}{l^{2}}\right)^{\frac{1}{2}}
$$

However, it is not clear whether the above distribution is a good approximation when compared to Persson's analytical solution (see Appendix 1).

We performed the finite element calculation (details in Appendix 3) to obtain the contact pressure $p_{y}$ distribution along the chord, and the comparison with Persson's solution and Hertz solution is shown in Figure 2. The finite element results for different compressive displacements $(\delta=0.5 \Delta R: 2 \Delta R)$ agree well with Persson's solution. The Hertz distribution used in literature ${ }^{15,16,20}$ deviates largely from both solutions (FEM and Persson's solution) as the location factor $x / l$ exceeds 0.5 .

We propose a parabolic formula to present the contact pressure distribution in terms of the semi-chord length $l$ and the maximum pressure $p_{0}$ at the center

$$
p_{y}=p_{0}\left(1-\frac{x^{2}}{l^{2}}\right)
$$

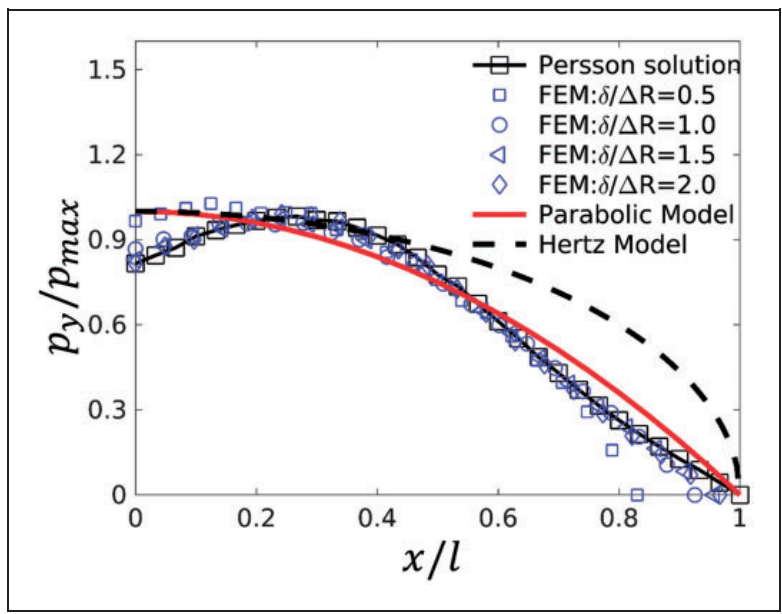

Figure 2. The distribution of the contact pressure $p_{y}$ along the chord by different models and FEM calculation.
It can be seen from Figure 2 that the parabolic distribution (solid line) agrees relatively well with FEM results and Persson's solution along the cord. Therefore, we will propose the explicit NFD relationship based on the parabolic distribution.

\section{NFD relation for elastic conformal contact}

Based on the previous analysis, the parabolic model is adopted to describe the contact pressure $p_{y}$ distribution along the chord, and then the NFD relation can be derived by combining the boundary conditions. The displacement boundary condition in the contact of cylindrical joints should satisfy the geometrical relationship (see Figure 1)

$$
u_{1}-u_{2}=\delta \cos \alpha-\left(R_{2}-R_{1}\right)(1-\cos \alpha)
$$

Here, $u_{1}$ and $u_{2}$ are radial displacements of the cylinder and the hole at the point of angle $\alpha$, respectively. $\delta$ is the compressive displacement. The semiangle of contact $\varepsilon$ is obtained by taking $u_{1}-u_{2}=0$; therefore, the semi-chord length $l$ and contact angle $\varepsilon$ can be expressed as

$$
l=2 R \sin \frac{\varepsilon}{2}, \quad \cos \varepsilon=\frac{\Delta R}{\Delta R+\delta}
$$

Here, small clearance (i.e. $R_{1}=\sim R_{2}$ ) assumption is taken, so that $R$ can take the value of $R_{2}$. The maximum pressure $p_{0}$ is determined from the Winkler model $^{21}$ as follows

$$
p_{0}=k \frac{E^{*} \delta}{R}
$$

where $k$ is a dimensionless coefficient required to be determined/fitted. Johnson model $^{8}$ also gives the maximum contact pressure $p_{0}$ as the function of the contact force as

$$
p_{0}=\left(\frac{2 E^{*} \cdot P}{\pi R}\right)^{\frac{1}{2}}
$$

where $E^{*}$ is the equivalent modulus and can be expressed as $1 / E^{*}=\left(1-v_{1}^{2}\right) / E_{1}+\left(1-v_{2}^{2}\right) / E_{2}$.

We carry out FEM simulations to study the maximum pressure $p_{0}$ under different compressive displacements. Comparison between the Winkler model and Johnson model is shown in Figure 3. The pressure in the Johnson model equation (7) is only consistent with the FEM results at small displacements, while the Winkler model matches well with the numerical results in a much broader range. By combining with the geometrical relationship in equation (5), an explicit solution for NFD is obtained for the cylindrical conformal contact. The external load is obtained by integrating the 


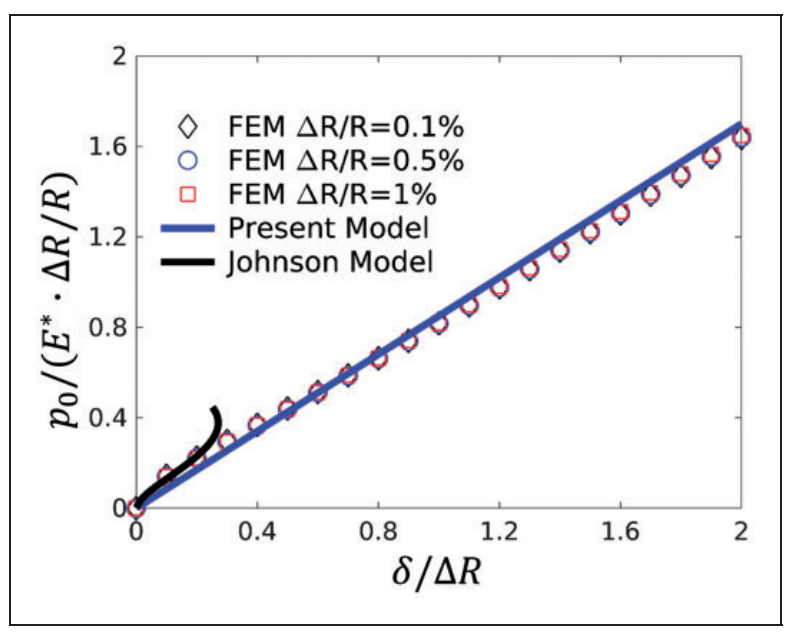

Figure 3. The maximum contact pressure as a function of the compressive displacement.

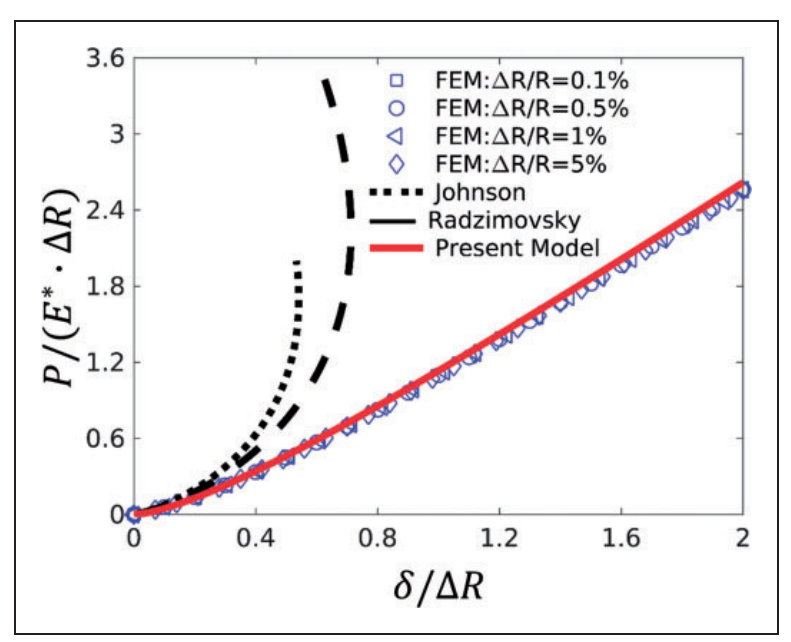

Figure 4. Normal force as a function of the compressive displacement.

parabolic distribution equation (3)

$$
P=2 \int_{0}^{l} p_{0}\left(1-\frac{x^{2}}{l^{2}}\right) \mathrm{d} x
$$

Using equations (5) and (6), the NFD can be obtained as

$$
P=\frac{8}{3} k E^{*} \delta \sqrt{\frac{\delta}{2(\Delta R+\delta)}}
$$

here, $k$ is fitted in Figure 3 with a value of 0.85 .

We compare our NFD relationship with the two commonly used models (Johnson ${ }^{8}$ and Radzimovsky, ${ }^{11}$ and model details can be found in Appendix 2). As shown in Figure 4, the NFD relations in these two models exhibit a parabolic relation between the load and displacement; this may lead to unphysical characteristics when the loading displacement/load is large. Thus, the load limit for Johnson and Radzimovsky models is approximately $1.71 E^{*} \Delta R$ and $2.41 E^{*} \Delta R$, respectively. Also, it can be seen from Figure 4 that the deviation of these two models from the FEM results becomes evident as the penetration displacement $\delta$ is larger than $0.5 \Delta R$. In contrast, the normal force evaluated by the proposed model equation (9) is consistent with the FEM results for the penetration up to $2 \Delta R$.

\section{An elasto-plastic contact model}

In the previous section, it has been shown that the proposed model can predict the NFD relation better than the classical models, especially in the high load range. However, for elasto-plastic material, plasticity will get involved in the contact problem even when the compressive displacement is small. The critical displacement $\delta^{0}$ when plasticity emerges can be obtained by the expression $\delta^{0} / R=\sigma_{Y} /\left(k E^{*}\right)$, where $\sigma_{Y}$ is the yield stress. The normalized loading displacement (strain) $\delta^{0} / R$ for traditional metals, e.g. copper, aluminum, and iron, is around $0.1 \%$ to $0.2 \%$ which is smaller than the deformation strain of joints in engineering; therefore, plasticity needs to be taken into account for the NFD relation of conformal contact.

Regarding the contact pressure (threshold value) in the plastic zone when considering perfect plasticity, Song et al. ${ }^{22}$ have shown that for asperity flattening (non-conformal contact), the contact pressure approaches the material hardness $3 \sigma_{Y}$. More recently, Ghaednia et al. ${ }^{23}$ in a review concluded that the ratio between the contact pressure and $\sigma_{Y}$ is not always around 3 , but varies according to the boundary conditions and deformation geometry. This ratio has not been reported for conformal contact so far. Therefore, we carried out FEM simulations with perfect plasticity, $\Delta R / R=0.5 \%$ and $\sigma_{Y} / E^{*}=0.2 \%$. The pressure is shown as a function of the distance to the contact center at a depth of $2 \Delta R$ in Figure 5(a). It is clearly seen that there is an apparent yield zone in the middle of the contact. Also, it is shown in Figure 5(b) for the conformal contact that the average contact pressure is around/slightly below than $\sigma_{Y}$, it is much less than the value of material hardness $3 \sigma_{Y}$ as in non-conformal contact. Therefore, for simplicity, we take the contact pressure to be $\sigma_{Y}$ in the plastic zone, as shown in Figure 6. We assume that the contact pressure distribution (solid line) still follows the parabolic distribution in the elastic contact zone. In the plastic zone (width of $2 a_{p}$ ), the parabolic contact pressure is truncated by a threshold $\sigma_{Y}$.

Similar to the purely elastic case, the external load can be obtained by integrating the pressure distribution as

$$
P=2 \int_{a_{p}}^{l} p_{0}\left(1-\frac{x^{2}}{l^{2}}\right) \mathrm{d} x+2 \sigma_{Y} a_{p}
$$

Here, $a_{p}$ can be calculated by $a_{p}=l \sqrt{1-\sigma_{Y} / p_{0}}$ and $p_{0}$ is given by the Winkler model as in equation (6). 


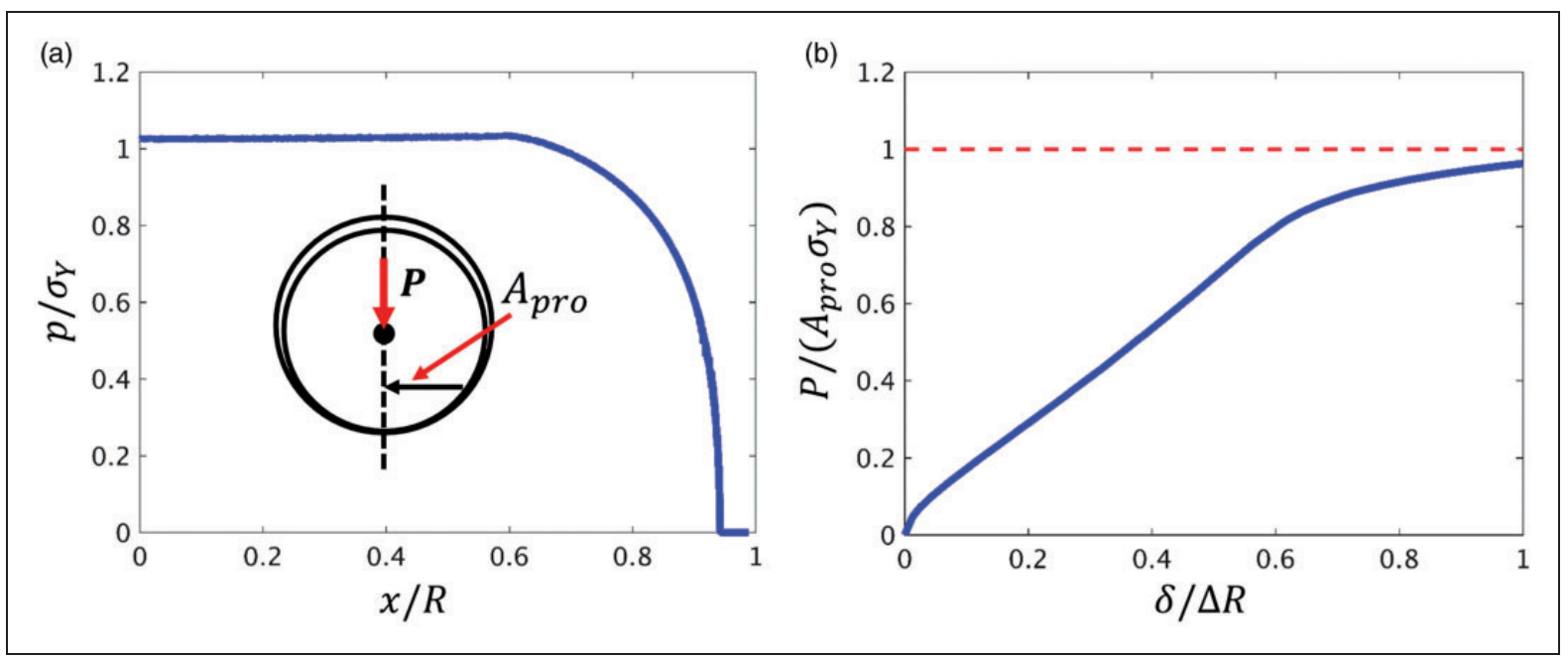

Figure 5. (a) Ratio of the local contact pressure to $\sigma_{Y}$ as a function of the distance to the contact center at the penetration depth of $2 \Delta R$. (b) Average contact pressure as a function of penetration depth. $P$ is the indentation force, $P / A_{\text {pro }}$ has the unit of pressure.

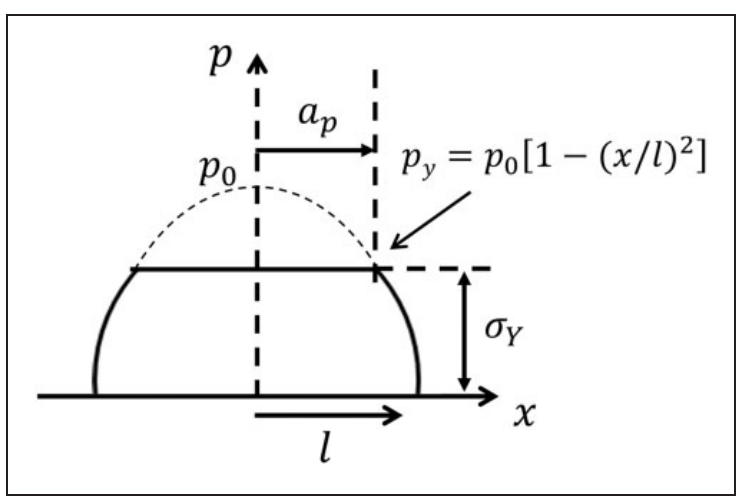

Figure 6. Profile of pressure distribution in the contact area considering plasticity.

From equations (5), (6), and (10), an explicit NFD relation is obtained

$$
\begin{aligned}
& \frac{P}{R \sigma_{Y}} \sqrt{2\left(\frac{\Delta R}{\delta}+1\right)} \\
& \quad= \begin{cases}\frac{4 \delta}{\delta^{0}}\left[\frac{2}{3}-\left(1-\frac{\delta^{0}}{\delta}\right)^{1 / 2}+\frac{1}{3}\left(1-\frac{\delta^{0}}{\delta}\right)^{3 / 2}\right]+4\left(1-\frac{\delta^{0}}{\delta}\right)^{1 / 2}, & \delta>\delta^{0} \\
\frac{8}{3} \cdot \frac{\delta}{\delta^{0}}, & \delta \leqslant \delta^{0}\end{cases}
\end{aligned}
$$

Compared to the elastic case with NFD expressed in equation (9), we can also write equation (11) in a more physical version; in the plastic region with penetration displacement $\delta$ is larger than $\sigma_{Y} R /\left(k E^{*}\right)$, the normal force can be expressed as

$$
\begin{aligned}
P= & \left\{k E^{*} \delta\left[\frac{2}{3}-\left(1-\frac{\sigma_{Y} R}{k E^{*} \delta}\right)^{1 / 2}+\frac{1}{3}\left(1-\frac{\sigma_{Y} R}{k E^{*} \delta}\right)^{3 / 2}\right]\right. \\
& \left.+R \sigma_{Y}\left(1-\frac{\sigma_{Y} R}{k E^{*} \delta}\right)^{1 / 2}\right\} \sqrt{\frac{8 \delta}{(\Delta R+\delta)}}
\end{aligned}
$$

In the elastic case, the normal force has a linear monotonic function of modulus $E^{*}$ as in equation (9). However, in the plastic case, the dependence of normal force on modulus $E^{*}$ is rather complicated as shown in equation (12). The dependence on yield strength $\sigma_{Y}$ is also not obvious.

We perform FEM calculations to validate the elastoplastic NFD relation in equation (11). In the FEM model, the perfect plasticity with the yield strength $\sigma_{Y}$ is used, and all the other parameters can be found in Appendix 3. The accuracy of the NFD model is verified by using different yield strengths, i.e. $\sigma_{Y} / E^{*}$ varies from $0.1 \%$ to $0.6 \%$. Different values of the clearance $(\Delta R / R=0.1 \%, 0.5 \%, 1.0 \%) \quad$ are also checked. It can be seen in Figure 7 that the NFD relation proposed here matches well with the FEM numerical results for different yield strengths and clearances.

For conformal contact, the transition of elastic to elasto-plastic contact is different from the transition in Hertz contact. In conformal contact, it can be seen in Figure 8 that the first yield occurs at the surface of the contact, as shown in left-down part of Figure 8. In Figure 8, the yield zone nucleated at "A", and the yielded area expands as the penetration displacement increases, as shown in " $\mathrm{B}$ " and " $\mathrm{C}$ ". The distribution of contact pressure at " $\mathrm{C}$ " is similar to the case as shown in Figure 5(a); it clearly shows a flat yield zone in the middle surrounded with the elastic zone. This further confirms the point that the pressure distribution can be physically represented by the plastic-Winkler model with profile as shown in Figure 6.

For the case of Hertz contact between an elasticperfectly plastic cylinder and a rigid flat, the yield point firstly appears in the position at a distance (about 0.7 times of the contact radius $^{8}$ ) below the contact surface. This transition is different from the conformal case, in which the first yielding occurs at the surface. And apparently, the plastic contact of 
non-conformal case cannot be represented by the plastic-Winkler model as shown in Figure 6.

The transition point predicted by the model can be calculated by giving correct value of $\frac{\sigma_{Y}}{E^{*}}$ and $\frac{\Delta R}{R}$, and in this case, the transition displacement is found to be $\delta_{0} / \Delta R=1.18$. It can be seen from finite element results in Figure 8 that the yield zone starts to appear after the

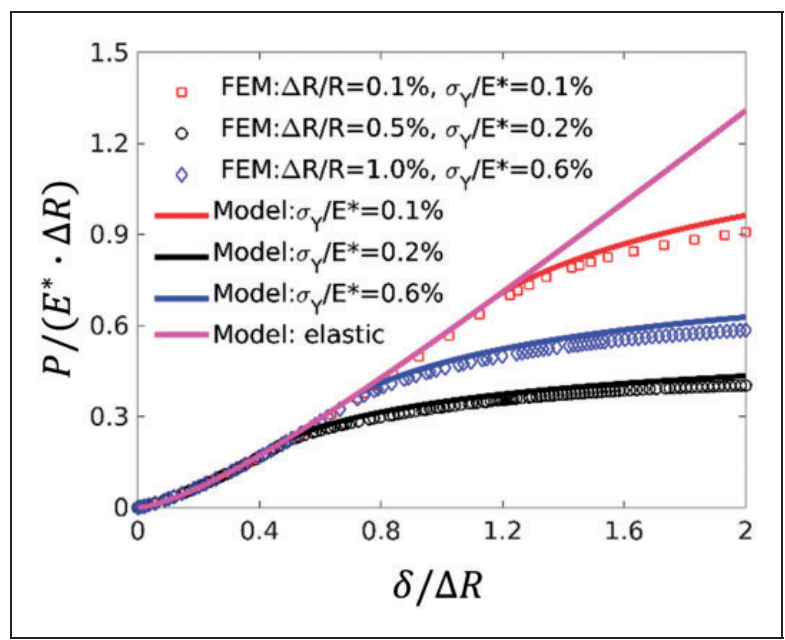

Figure 7. Normal force as a function of the compressive displacement for elasto-plastic contact. displacement $\delta / \Delta R$ is larger than 1.18. This point is also consistent with the branch between elastic and elasto-plastic curves in Figure 8.

Furthermore, the normal force is also studied as a function of the semi-angle of contact as shown in Figure 9, where Persson model (equation (13)) is also presented. It can be seen that our model agrees

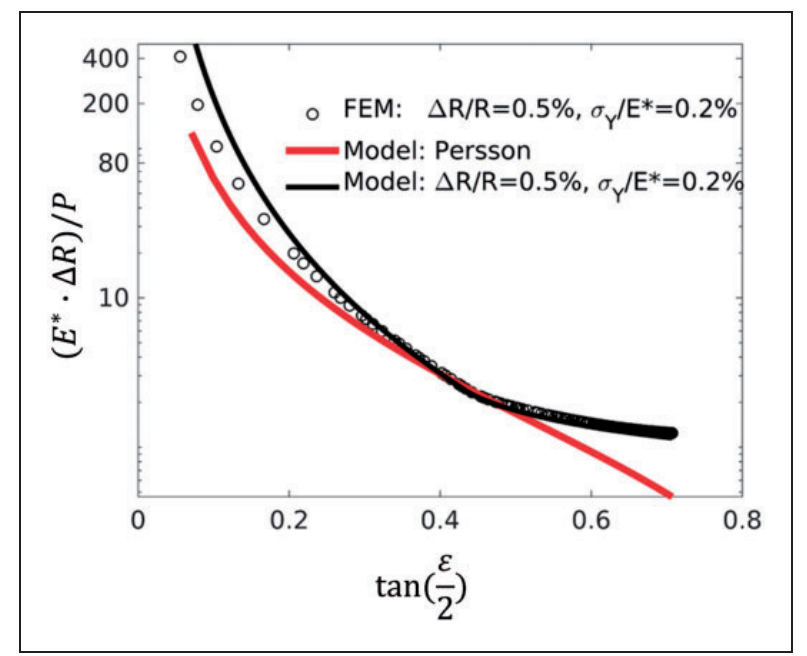

Figure 9. Relationship between the normal force and the contact angle.

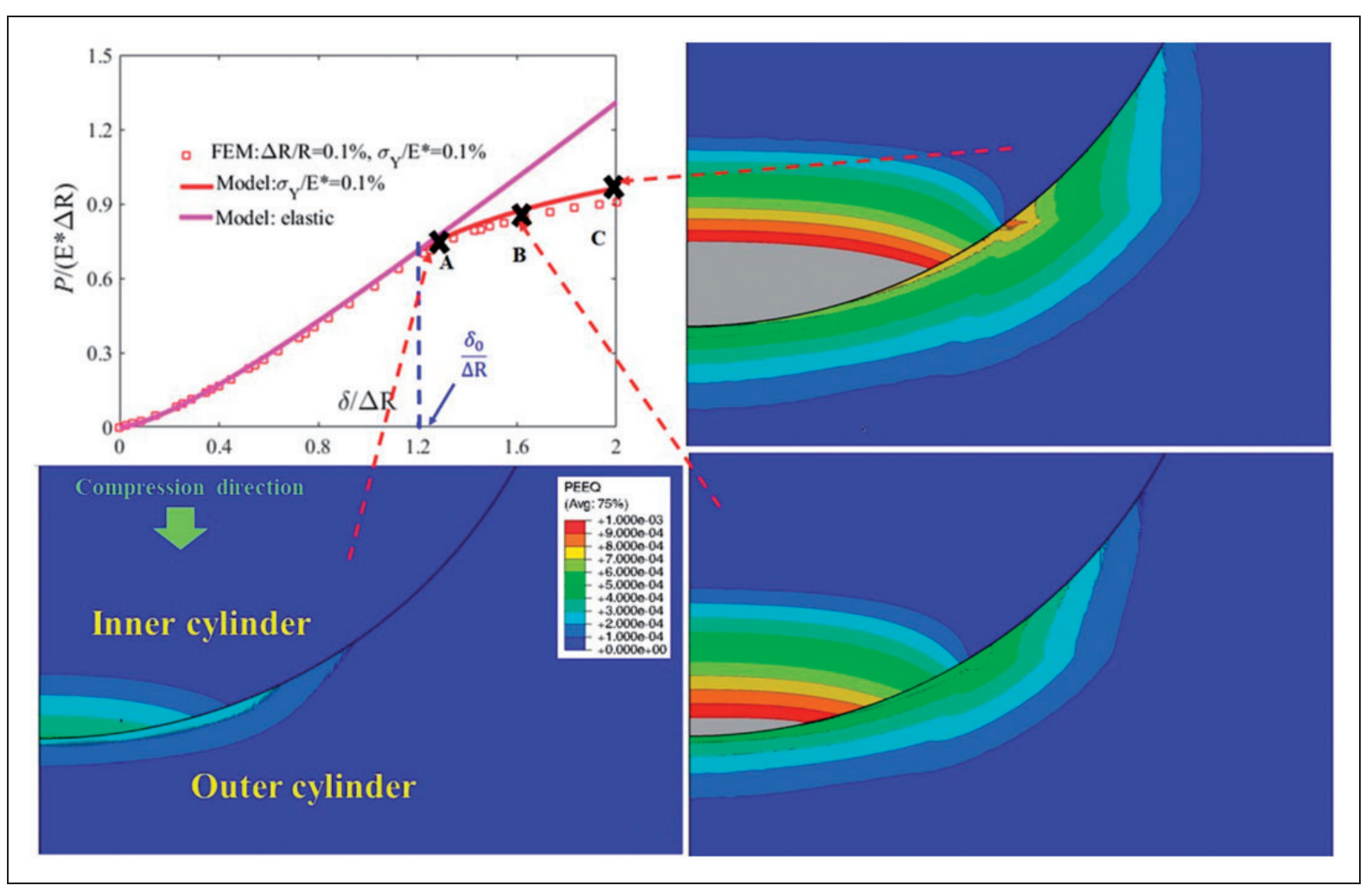

Figure 8. The elasto-plastic transition in conformal contact $\left(\Delta R / R=0.1 \%, \sigma_{Y} E^{*}=0.1 \%\right)$. The black crosses "A", "B", "C" in the left upper figure corresponding to the different stages of contact as shown in the rest figures. The distribution of equivalent plastic strain is shown here to indicate evolution of plastic zone. The legend equivalent plastic strain (PEEQ) is equivalent to the plastic strain, the yield zone is indicated by the area with PEEQ large than zero. The transition displacement predicted by the model is $\delta / \Delta R=I .18$. 
well with Persson's solution and FEM results in the elastic regime $\left(\tan \left(\frac{\varepsilon}{2}\right)<0.4\right)$. However, Persson model is not able to predict the normal force in the plastic regime, while our model matches the FEM results very well in the elasto-plastic regime.

\section{Conclusions}

In this paper, we introduced a parabolic contact pressure distribution for the conformal contact between cylindrical joints; the parabolic assumption matches well with Persson's solution and FEM simulation results. Through the Winkler model, we obtain an explicit formula of the NFD relationship. The obtained NFD relationship is compared with two conventional contact models for elastic deformation (i.e. Johnson model and Radzimovsky model). The comparison shows that the NFD relations predicted by the two models have a clear load limit which hinders the application, while our model matches the FEM results very well within the load range considered in this work. By using finite element simulation, it is observed that the transition of elasto-plastic contact in conformal case is different from the transition in Hertz contact. The plastic contact of conformal case can be physically represented by the plastic-Winkler model. Based on this assumption considering perfect plasticity, we also extend our contact model to the elasto-plastic regime and provide an explicit NFD relationship. The proposed NFD relationship matches FEM simulation results well for various material parameters and loadings.

\section{Declaration of Conflicting Interests}

The author(s) declared no potential conflicts of interest with respect to the research, authorship, and/or publication of this article.

\section{Funding}

The author(s) disclosed receipt of the following financial support for the research, authorship, and/or publication of this article: The work is supported by the National Natural Science Foundation of China (Grant Nos. 11772334, 11802310, 11672301), and National Key R\&D Program of China (No. 2017YFA0204402). The work is also supported by the Youth Innovation Promotion Association CAS (2018022) and by the Strategic Priority Research Program of the Chinese Academy of Sciences, Grant No. XDB22040501.

\section{ORCID iD}

Xiaoming Liu (D) https://orcid.org/0000-0003-0235-1894

\section{References}

1. Flores P and Ambrósio J. Revolute joints with clearance in multibody systems. Comput Struct 2004; 82: 1359-1369.

2. Ciavarella M, Baldini A, Barber JR, et al. Reduced dependence on loading parameters in almost conforming contacts. Int J Mech Sci 2006; 48: 917-925.
3. Pereira C, Ambrósio J and Ramalho A. Contact mechanics in cylindrical clearance revolute joints. Appl Mech Mater 2013; 365-366: 412-415.

4. Persson A. On the stress distribution of cylindrical elastic bodies in contact. PhD Thesis, Chalmers University of Technology, 1964.

5. Ciavarella M and Decuzzi P. The state of stress induced by the plane frictionless cylindrical contact. I. The case of elastic similarity. Int J Solids Struct 2001; 38: 4507-4523.

6. Ciavarella M and Decuzzi P. The state of stress induced by the plane frictionless cylindrical contact. II. The general case (elastic dissimilarity). Int J Solids Struct 2001; 38: 4525-4533.

7. Fang X, Zhang C, Chen X, et al. A new universal approximate model for conformal contact and non-conformal contact of spherical surfaces. Acta Mech 2014; 226: 1657-1672.

8. Johnson KL. Contact mechanics. Cambridge: Cambridge University Press, 1985.

9. Lankarani HM and Nikravesh PE. A contact force model with hysteresis damping for impact analysis of multibody systems. J Mech Des 1990; 112: 369-376.

10. Zhu A, He S, Zhao J, et al. A nonlinear contact pressure distribution model for wear calculation of planar revolute joint with clearance. Nonlinear Dyn 2016; 88: 315-328.

11. Radzimovsky EI. Stress distribution and strength conditions of two rolling cylinders pressed together. Champaign: University of Illinois Press, 1953, p.408.

12. Pereira CM, Ramalho AL and Ambrósio JA. A critical overview of internal and external cylinder contact force models. Nonlinear Dyn 2010; 63: 681-697.

13. Pereira CM, Ramalho AL and Ambrósio JA. Applicability domain of internal cylindrical contact force models. Mech Mach Theory 2014; 78: 141-157.

14. Pereira CM, Ramalho AL and Ambrósio JA. An enhanced cylindrical contact force model. Multibody Syst Dyn 2015; 35: 277-298.

15. Liu C, Zhang K and Yang L. The compliance contact model of cylindrical joints with clearances. Acta Mech Sin 2005; 21: 451-458.

16. Liu $\mathrm{C}$, Zhang $\mathrm{K}$ and Yang R. The FEM analysis and approximate model for cylindrical joints with clearances. Mech Mach Theory 2007; 42: 183-197.

17. Blanco-Lorenzo J, Santamaria J, Vadillo EG, et al. A contact mechanics study of $3 \mathrm{D}$ frictional conformal contact. Tribol Int 2018; 119: 143-156.

18. Knight MG, de Lacerda LA, Wrobel LC, et al. Parametric study of the contact stresses around spherical and cylindrical inclusions. Comput Mater Sci 2002; 25: $115-121$.

19. Noble B and Hussain MA. Exact solution of certain dual series for indentation and inclusion problems. Int J Eng Sci 1969; 7: 1149-1161.

20. Liu C, Zhang K and Yang L. Normal force-displacement relationship of spherical joints with clearances. J Comput Nonlinear Dyn 2006; 1: 160-167.

21. Perez-Gonzalez A, Fenollosa-Esteve C, Sancho-Bru JL, et al. A modified elastic foundation contact model for application in 3D models of the prosthetic knee. Med Eng Phys 2008; 30: 387-398.

22. Song H, Van der Giessen E and Liu X. Strain gradient plasticity analysis of elasto-plastic contact between rough surfaces. J Mech Phys Solids 2016; 96: 18-28. 
23. Ghaednia H, Wang X, Saha S, et al. A review of elasticplastic contact mechanics. Appl Mech Rev 2017; 69: 060804.

24. ABAQUS. Abaqus user's manual. USA: ABAQUS, 2014.

\section{Appendix}

\section{Notation}

$E_{1} \quad$ Young's modulus of the inner cylinder $E_{2} \quad$ Young's modulus of the outer cylinder

$l \quad$ semi-chord length corresponding to the

$R_{1} \quad$ radius of the internal cylinder

$R_{2} \quad$ radius of the outer circular hole

$\alpha \quad$ angle from the points on the interface

departure from the centric axis

$\delta \quad$ compressive displacement

$\delta^{0} \quad$ critical penetration when plasticity

$\Delta R \quad$ radial clearance of joints, $\Delta R=R_{2}-R_{1}$

$\varepsilon \quad$ semi-angle of contact corresponding to the whole contact arc

$v_{1} \quad$ Poisson's ratio for the inner cylinder

$v_{2} \quad$ Poisson's ratio for the outer cylinder

\section{Appendix I. Persson's solution}

References ${ }^{5,6}$ provide a completely closed form solution (i.e. Persson's solution) for the contact problem of cylindrical joints, in which the contact pressure has been derived as a function of the contact angle $\alpha$

$$
\begin{aligned}
\frac{R \cdot p(t)}{P}= & \frac{2}{\pi \sqrt{b^{2}+1}} \frac{\sqrt{b^{2}-t^{2}}}{1+t^{2}} \\
& +\frac{1}{2 \pi b^{2}\left(1+b^{2}\right)} \log \left(\frac{\sqrt{b^{2}+1}+\sqrt{b^{2}-t^{2}}}{\sqrt{b^{2}+1}-\sqrt{b^{2}-t^{2}}}\right)
\end{aligned}
$$

Here, $P$ is the external load and $R$ is the curvature of the contact surface. $b$ and $t$ are the contact parameters and expressed as

$$
b=\tan \left(\frac{\varepsilon}{2}\right), \quad t=\tan \left(\frac{\alpha}{2}\right)
$$

Then the projection of the pressure in the $y$-direction as shown in Figure 1 can be expressed as

$$
p_{y}=\frac{p(t) \cdot \cos \alpha \cdot \cos ^{2}\left(\frac{\varepsilon}{2}-\alpha\right)}{\cos \left(\frac{\varepsilon}{2}\right)}
$$

\section{Appendix 2. Johnson and Radzimovsky contact model}

Two NFD models proposed by Johnson and Radzimovsky are presented here, respectively. Based on the Hertz theory, Johnson ${ }^{8}$ developed a contact model in which the relationship between the compression displacement $\delta$ and the external load $P$ can be calculated by

$$
\delta=\frac{P}{\pi E^{*}}\left[\ln \left(\frac{4 \pi E^{*} \Delta R}{P}\right)-1\right]
$$

For the contact between external cylinders, formulas from Radzimovsky ${ }^{11}$ have been used to derive the NFD relationship

$$
\delta=\frac{P}{\pi E^{*}}\left[\frac{2}{3}+\ln \left(\frac{4 R_{1}}{d}\right)+\ln \left(\frac{4 R_{2}}{d}\right)\right]
$$

Here, for cylinders with different elastic material properties, the parameter $d$ in equation (17) is evaluated by

$$
d=1.6\left(\frac{R^{*} P}{E^{*}}\right)^{\frac{1}{2}}
$$

where $R^{*}$ is the relative curvature of conformal contact and is calculated by

$$
R^{*}=\frac{R_{1} R_{2}}{\Delta R}
$$

\section{Appendix 3. FEM simulations}

FEM simulations are carried out to obtain the contact pressure distribution in the contact of cylindrical joints with small clearances. The contact force distribution on the chord is calculated by projecting the contact pressure from the circular arc to the chord. 


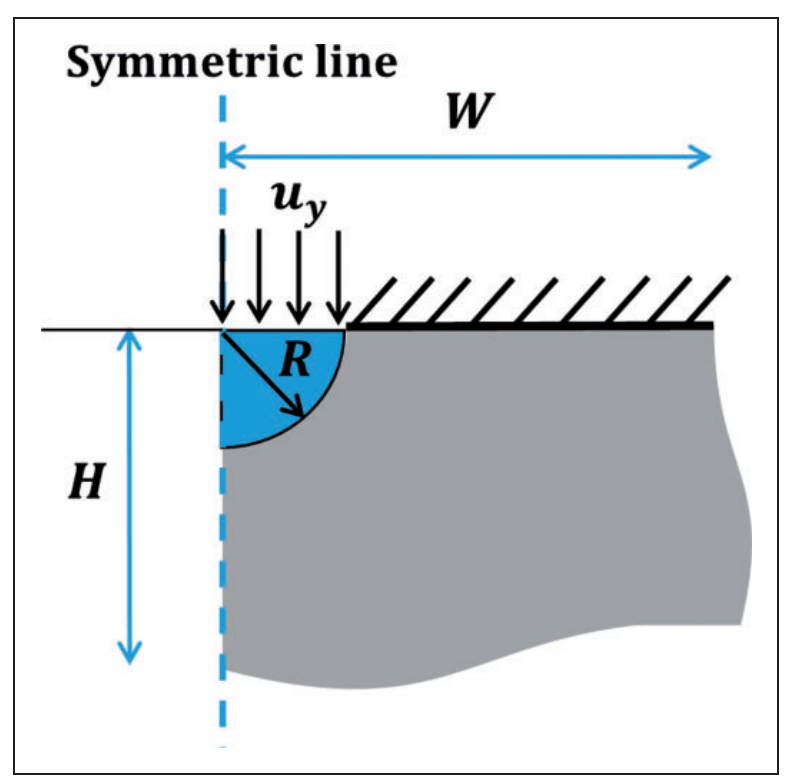

Figure 10. FEM model for cylindrical joints with clearance.

The FEM numerical model is performed by the commercial software ABAQUS. ${ }^{24}$ The inner cylinder has a radius $R$, and the radius of the hole is set as $R+\Delta R$. Due to the symmetry of the cylindrical joints, a quarter is modeled as shown in Figure 10. The symmetric boundary is applied on the middle dotted line. The top surface of the outer body is fixed, and the inner cylinder is displaced with fixed displacement $u_{y}$ in the vertical direction from the top. The dimensions of the outer body in the horizontal direction and

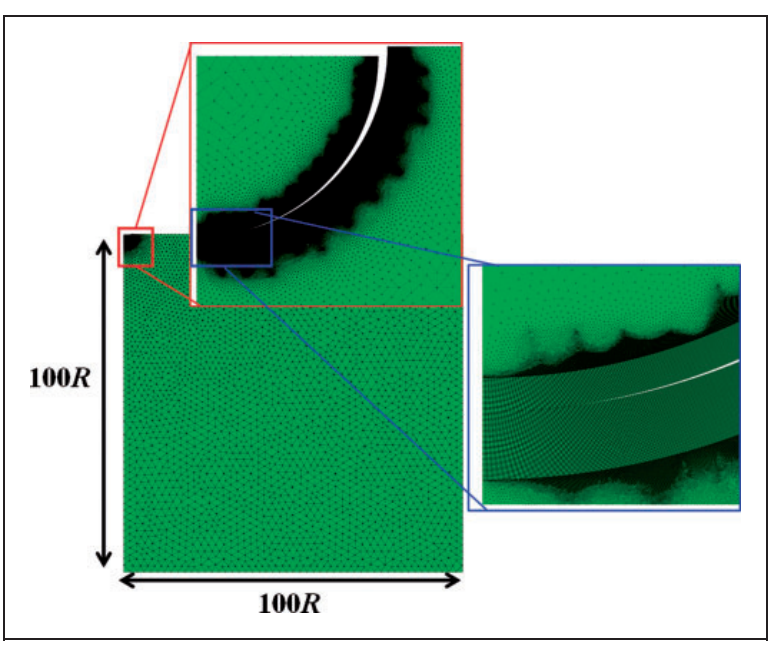

Figure I I. FEM mesh for the simulation (element size in the contact zone is set to be $0.1 \% R$ ).

vertical direction (defined as $W$ and $H$, respectively) are set to $100 R$ in order to eliminate the boundary effect. The bottom and the right side of the outer body in Figure 10 are set as free. The mesh in the FEM model, as shown in Figure 11, is applied with suitable element size in the contact area between the cylinder and hole. Plane strain element is used to simulate infinite long cylinders. The mesh size in the cylinder is in gradient distribution. Element dimensions with $1 \% R$ and $0.1 \% R$ are both tested, and the results show negligible difference. Thus, element size is set to $0.1 \% R$ in the subsequent FEM calculations. 